\title{
Prevalence of minority Human Immunodeficiency virus multi-drug resistant mutations among patients failing a Nucleoside Reverse Transcriptase Inhibitor based regimen in Uganda
}

Immaculate Lillian Nankya ( $\Delta$ inankya@jcrc.org.ug)

Joint Clinical Research Center

Eva Nabulime

Joint clinical Research Center

Fred Kyeyune

Joint clinical Research Center

Cissy Mutuluza Kityo

Joint clinical Research Center

Miguel Mateu Quinones

Case Western Reserve University

\section{Research}

Keywords: HIV genotyping, Multi-Drug Resistance, Next Generation Sequencing, minority variants

Posted Date: July 26th, 2021

DOI: https://doi.org/10.21203/rs.3.rs-666492/v1

License: (c) (i) This work is licensed under a Creative Commons Attribution 4.0 International License.

Read Full License 


\section{Abstract \\ Objective}

To determine the prevalence of multi-drug resistant variants among patients failing on a nucleoside reverse transcriptase inhibitor (NRTI) based regimen with a detectable viral load $\geq 1000$ copies $/ \mathrm{ml}$ among patients harboring HIV subtype $A, C$ and $D$.

\section{Methods}

Samples were obtained from patients who were failing on an NRTI based regimen. Sanger based sequencing was performed as part of the standard of care. Mutation analysis was performed using the Stanford HIV drug Resistance database. A subset of these patient samples was further analyzed using the Next Generation Sequencing (NGS) technology and analysis of the drug resistance mutations was performed at the $20 \%$ and $1 \%$ cut off

\section{Results}

Analysis of the Non-nucleoside reverse transcriptase inhibitor (NNRTI) coding region revealed that the K101 and the Y181 mutations were more predominant among subtype $C$ than subtype $A$ and $D$. Although Thymidine analog mutations (TAMs) were prevalent in all subtypes, our analyses showed that these mutations occurred in significantly less proportions among subtype $C$ infections when compared with the subtype A and D counterparts. Furthermore, the Q151M mutation complex which involves mutations in multiple domains was significantly more prominent among patients harboring subtype $C$ variants. Analysis using NGS revealed that minority drug resistant mutations that confer multi-drug resistance (MDR) were present even in patients who exhibited a susceptible genotype based on the Sanger sequencing technique.

\section{Conclusion}

Although HIV-1 MDR variants occur in all subtypes, their predominance is subtype specific with TAMs being significantly more predominant among subtype A and D while the Q151M complex being significantly more predominant among patients harboring subtype $C$ viruses. Even in patients with a susceptible genotype based on Sanger technology, minority variants are present and their evolution to full blown MDR occurs over time such that by the time they are detectable, cross resistance to other drugs has occurred in some cases.

\section{Background}


It is almost twenty years since the global roll out of Anti-retroviral therapy (ART) and according the UNAIDS report 2020, of the 37.9 million individuals currently living with HIV, $63 \%$ have been initiated on ART. Although we are still lagging behind the 2020 UNAIDS target [1-4], it is undeniable that ART has tremendously changed the face of the Human Immunodeficiency Virus (HIV) epidemic resulting in a marked reduction in HIV associated mortality and a significant reduction in opportunistic infections, therefore, more people are living prolonged and more productive lives [5, 6]. The current 90-90-90 strategy, ensures that all the patients who are initiated on ART are closely monitored so that they achieve viral suppression [7-11]. This has a twofold advantage: it ensures that individuals are living more healthy lives and also it reduces the transmission rates to almost zero $[12,13]$. Along with the 90-90-90 treatment strategy, WHO currently recommends the Test and Treat approach implying that for the second 90, treatment is initiated as soon as a positive HIV test is obtained [14-22]. However, it is important to note that although the first and second 90 have been achieved in most of the countries, the third 90 is still lagging behind; yet in order to reduce viral transmission, sustained viral suppression is a paramount [23, 24]. Furthermore, the 90-90-90 treatment approach was started a few years ago implying that the mature cohorts of patients who have been on ART for a long time may not have got the much needed monitoring and this may inevitably spill into the younger cohorts who were initiated during this current Test and Treat era thereby further affecting the attainment of the third 90 .

The ability for HIV to develop drug resistance mutations is a major obstacle to long-term effective antiHIV therapy [25-28]. In order to achieve complete viral suppression, there is need to maintain consistently elevated drug levels within an individual. However, in most individuals, this is not possible for a number of reasons: poor adherence, incorrect drug dose, drug interactions, and poor absorption among others [29-35]. This results in insufficient drug levels within an individual which then enable the virus to replicate under these suboptimal drug conditions. The emerging virus is one that is able to escape drug pressure and hence it is the drug resistant variants that emerge. This then results in the regimen being ineffective. The increased clinical use of combination antiretroviral treatment for HIV-1 infection has led to the selection of viral strains resistant to multiple drugs, including strains resistant to all licensed nucleoside analog RT inhibitors and protease inhibitors [28, 36-42]. Although research continues to improve our understanding of drug resistance leading to refined treatment strategies and, in some cases, improved outcome, resistance to antiretroviral therapy remains a major cause of treatment failure among patients living with HIV-1 [43-45].

Since drug resistant mutations evolve over time, it is paramount that they are detected much early in their course to prevent the emergence of multi-drug resistant mutations which are a huge challenge especially in the low resource settings where limited treatment options prevail [46-49]. Three drug resistance patterns confer multi-drug resistance namely: thymidine analogue-associated mutations (TAMs), the 69 Insertion (69lns) and the Q151M complex. TAMs, originally selected by the thymidine analogues zidovudine (AZT) and stavudine (d4T), also confer reduced susceptibility to all approved NRTIs, depending on the number and type of mutations involved [50-53]. There are two TAM pathways: type I (M41L, L210W and T215Y) [54, 55] and type II (D67N, K70R, T215F and K219Q/E) [55]; the former conferring higher levels of resistance and cross-resistance. TAMS are commonly found in low-income 
countries where thymidine analogue-containing regimens have been prescribed as first line ART [56, 57]. It is important to note that although Tenofovir (TDF) is the preferred drug in most first line regimens, AZT is still being prescribed as an alternative. Furthermore, there has been documented antagonism between TDF and TAMs [51, 52]. The 69 Ins consists of a substitution at codon 69 and an insertion of two or more amino acids [58-63]. When present with at least one TAM at position 41, 210 or 215, resistance to all approved NRTIs is conferred [64]. Q151M is usually accompanied by the mutations A62V, V75I, F77L and F116Y [65]. The Q151M complex affects all approved NRTIs except for tenofovir [65]. M184V is another important mutation which is also the most commonly occurring NRTI resistance associated mutations (RAM). M184V causes high-level resistance to lamivudine and emtricitabine and low-level resistance to didanosine and abacavir [66-69]. When present with TAMS, M184V can increase resistance to abacavir. The presence of TAMs, 69Ins, Q151M and M184V at first-line ART failure can compromise treatment options for second-line therapy.

Since the global roll out of ART, we have cohorts that have matured and prior to the 90-90-90 most of the patients in low resource settings were not receiving the required close monitoring. This meant that individuals were kept on a failing regimen for quite some time and this inevitably resulted in the emergence of multi-drug resistance. This kind of resistance is cross cutting affecting all the drug NRTI drug classes. For this study, we retrospectively analyzed samples from patients who had been on various first line regimens for the presence of multi-drug resistance mutations. We used next generation sequencing (NGS) using the lon Torrent platform to determine the frequencies of drug resistance mutations that are associated with multi-drug resistance. All these samples had been sequenced using the Sanger sequencing platform which has a limit of detection of $20 \%$ implying that if a mutation is present at less than $20 \%$ of the population, it is not readily detected. For both Sanger sequencing and NGS, we assayed for the following mutations Thymidine analog mutations (TAMS) and these include: type I (M41L, L210W and T215Y) and type II (D67N, K70R, T215F and K219Q/E); the Q151 mutation complex which includes the following mutations: A62V, V75I, F77L and F116Y and the 69ins complex.

\section{Materials And Methods}

\section{Study population}

The Joint Clinical Research Centre (JCRC) is an HIV care and research center that has been offering antiretroviral therapy to HIV positive patients for close to thirty years. In the early 1990s, although treatment was offered, it was suboptimal, mainly mono and dual therapy. Triple therapy was introduced in the late 90 s but still it was not readily available. With the global roll out of ART in 2004, treatment became universally available to all patients with the first line regimen comprising of two NRT Is plus a non-nucleoside reverse transcriptase inhibitor (NNRTI). Patients were able to have full access to virological monitoring in order to determine response to therapy and this included a viral load test as well as a drug resistance test for those with a viral load greater than 1000 copies $/ \mathrm{ml}$. Ethical clearance for this analysis was provided by the institutional review board (protocol, EM-10-07) at JCRC, Kampala Uganda. 


\section{Clinical Samples}

Using the patient care database, all patients failing on a Reverse Transcriptase (RT) based regimen as evidenced by a viral load greater than $1000 \mathrm{copies} / \mathrm{ml}$ were selected. For a sample to be included in the analysis, it had to have an HIV genotype result in the reverse transcriptase region based on Sanger sequencing technology. Anonymized patient databases containing patient demographics and laboratory results were merged with HIV-1 drug resistance databases after stripping samples of identifiers in accordance with the IRB approval.

\section{RNA extraction and PCR amplification}

Viral RNA was extracted from 400 plasma samples using a QIAamp viral RNA Mini Kit (Qiagen) according to the manufacturer's instructions. Reverse transcription of the HIV-1 reverse transcriptase (RT) coding region from extracted viral RNA and amplification was done with the sense primer PS2 (5'-

TCCCTCAAATCACTCTTTGGCAAC-3') and antisense primer RTA8 (5'-

GCTATTAAGTCTTTTGATGGGTCATT-3') using a Superscript III single RT-PCR system with Platinum Taq DNA polymerase kit (Thermo Fisher scientific) as per the manufacturer's instructions. Nested PCR was done using the sense primer RTS1AD (5'- CTGTACCAGTAAAATTAAAGCCAGG-3') and antisense RTA4 (5'CTGTATATCATTGACAGTCCAGCT-3') using a Platinum Taq kit (Thermo Fisher Scientific) as per the manufacturer's instructions to generate amplicon of 800 base pairs. The amplicon was purified using ExoSAP-IT enzyme (Thermo Fisher Scientific) and quantified using a Qubit fluorometer (Thermo Fisher Scientific).

Genetic analysis by Sanger sequencing: Data analysis was performed on already sequenced patient samples that were sequenced on the $\mathrm{ABI} 3730 \mathrm{xl}$ genetic analyzer and mutation calling performed using the Genotypic Resistance Interpretation Algorithm from the Stanford University HIV Drug Resistance Database (http://hivdb.stanford.edu) to infer the levels of susceptibility to the reverse transcriptase coding region.

\section{Genetic analysis by Next Generation Sequencing (NGS)}

PCR products encompassing the reverse transcriptase (RT) coding region of the HIV genome were sequenced using Deegen as described by Gibson et al [70]. Briefly, amplicons were purified (Agencourt AMPure XP, Beckman Coulter) and quantified (Bioanalyzer DNA 7500, Agilent Technologies) prior to using the Ion Xpress Fragment Library Kit (Life Technologies, Carlsbad CA) to construct a multiplexed library for shotgun sequencing on the lon Personal Genome Machine (PGM, Life Technologies). For that, 33ng of purified DNA amplicons was randomly fragmented and blunt-ends repaired using the lon Shear Plus Reagent (Life Technologies) followed by DNA purification (Agencourt AMPure XP, Beckman Coulter). The P1 adapter and one of 12 barcodes were ligated to the repaired fragment ends prior to DNA purification (Agencourt AMPure XP, Beckman Coulter). DNA fragments were then selected by size (i.e., 280 to 320 bp; Pippin Prep, Life Technologies) and each barcoded library, was purified (Agencourt AMPure XP, Beckman Coulter) and normalized using the Ion Library Equalizer Kit (Life Technologies). All thirteen barcoded DNA 
libraries, corresponding to the patient-derived amplicons plus the HIV-1NL4-3 control, were pooled in equimolar concentrations and templates prepared and enriched for sequencing on the lon Sphere Particles (ISPs) using the lon OneTouch 200 Template Kit v2 (Life Technologies) in the lon OneTouch 2 System (Life Technologies). Templated ISPs were quantified (Qubit 2.0, Life Technologies) and loaded into an Ion 316 Chip (Life Technologies) to be sequenced on the lon PGM using the lon PGM Sequencing 200 Kit v2 (Life Technologies). Following a 4 hour and 20 minutes sequencing run, signal processing and base calling was performed with Torrent Analysis Suite version 3.4.2.

Read mapping and variant calling: Reads were mapped and aligned against sample-specific reference sequences constructed for the reverse transcriptase region using the DEEPGEN Software Tool Suite. The frequency of each amino acid present in each HIV-1 genomic position was calculated and summarized in a graphical interface with particular focus on sites of known drug resistance based on the latest edition of the IAS-USA HIV Drug Resistance Mutations list [57]. A list of the amino acids at these positions, and their frequencies, was exported as a tabulated text file and used with the HIVdb Program Genotypic Resistance Interpretation Algorithm from the Stanford University HIV Drug Resistance Database (http://hivdb.stanford.edu) to infer the levels of susceptibility to reverse transcriptase inhibitors.

\section{Results}

Based on the sequence analysis of the RT coding region, subtype A is the most predominant subtype, followed by subtype $D$ then $C$ (Fig. 1). Our findings are in line with what other studies have previously found.

The dataset we analyzed was comprised of patients harboring subtype A, C and D HIV-1 viruses. First, we analyzed for mutations that confer resistance to the NNRTI class of drugs. A comparison was done by subtype in order to find out if we were in agreement with the already documented mutation differences. In this analysis we found that although $\mathrm{K} 101$ and $\mathrm{Y} 181$ occurred among subtype A and D patients, they were found in greater proportions among subtype C patients (Fig. 2A). Of note was the V106 mutation which was almost absent among subtype $D$ patients but was found in significantly higher proportions among subtype $C$ patients (Fig. 2A). Although there were notable subtype specific differences, overall, subtype A patients harbored the highest proportion of mutations that confer resistance to the NNRTI class of drugs, this was followed by subtype C (Fig. 2B).

Next, we analyzed for the occurrence of Thymidine Analog Mutations (TAMS) among these three subtypes. While the difference in occurrence of these mutations were not significantly different between subtypes $A$ and $D$, it was very evident that although these mutations occurred in subtype $C$, their proportions in this subtype were much lower than what was seen in subtypes $A$ and $D$ (Fig. $3 A$ ). When we looked at the overall occurrence of TAMs among these subtypes, subtype $A$ harbored these mutations in the highest proportions (Fig. 3B).

Since the Q151 complex is known for causing multidrug resistance which affects all NRTI drugs except Tenofovir, we analyzed for this mutation complex among the three subtypes. Apart from V75, the rest of 
the mutations within this complex were almost undetectable among subtype $D$ patients. On the other hand, every one of these mutations was found in significantly higher proportions among patients harboring subtype $\mathrm{C}$ viruses (Fig. $4 \mathrm{~A}$ ). Overall analysis of the mutations in this complex ranked subtype $\mathrm{C}$ with the highest proportion while subtype D had the lowest proportion (Fig. 4B).

All samples analyzed were from patients who had presumably failed on a Reverse Transcriptase Inhibitor (RTI) based regimen as evidenced by a viral load greater than 1000 copies $/ \mathrm{ml}$. Using the Sanger based sequencing technique, these patient samples were classified into two, those that exhibited drug resistant mutations in the RT coding region and those that did not exhibit any drug resistant mutations to RTIs. These two groups of samples were then sequenced on the lon Torrent NGS platform and analyzed for the presence of mutations that confer drug resistance mutation to the RT coding region. A general analysis was done on the sequence data obtained from NGS. Analysis at each of the codons targeted by NRTIs and NNRTIs for patient samples that exhibited mutations on the Sanger based technology, we found that for the majority of these patients in addition to detection of mutations at high frequencies, other mutations that confer drug resistance to NRTIs were detected at much lower frequencies below the $20 \%$ detection limit for Sanger sequencing (Fig. 5A). When we performed the same analysis on the samples from patients who did not exhibit any drug resistance mutations to the NRT Is and NNRTIs, we found that although the high mutation frequencies at each of these codons were not detectable, the proportions of patients exhibiting low level frequency mutations at each of these positions was high (Fig. 5B).

Next, for these two groups of patient samples, we analyzed for the presence of TAMs occurring at more $20 \%$ of the viral population within individuals. This is the viral population that is also detected by the Sanger Sequencing technology. We found that the results we obtained from NGS were comparable to what was obtained from Sanger (Data not shown). For the minority variants occurring at drug resistant mutation sites that are associated with Thymidine analogs, we found that at least $5 \%$ of the patients failing an NRTIs based regimen with any of the TAMs based on Sanger sequencing harbored TAMS at other sites but at levels less than $20 \%$ of the viral population and this was only detected by the use of the NGS sequencing technology (Fig. 6A). On analyzing the samples obtained from patients who did not exhibit any drug resistant mutations based on Sanger sequencing, we found that although these patients did not show any TAMs at a level greater than $20 \%$ (which is also the reportable range for Sanger sequencing), minority variants conferring resistance to thymidine analogs were readily detected in varying proportions among these patients (Fig. 6B).

Finally, we analyzed for the Q151 complex in in these two groups of patients. In agreement with Sanger sequencing technology, this complex although detectable at a much lower frequency within the patient papulation, it was well detected at levels comparable to those obtained with Sanger sequencing (Fig. 7A). In addition, using NGS, we were able to detect mutations within the Q151 complex although these were occurring at much lower proportions within the patients when compared to TAMs (Fig. 7B).

\section{Discussion}


Test and Treat, the recommended treatment strategy for the initiation of ART in HIV positive individuals is done in such a way that once a patient reports to the health facility for testing, they are initiated on ART the same day a positive result is obtained. This has the advantage of ensuring that no one is lost to follow up prior to initiation of therapy. The disadvantage of the Test and Treat approach however is the fact that patients are initiated on therapy without performing a baseline drug resistance test which in the developed countries guides to the best regimen the patient can be started on. Furthermore, these developed countries have long switched from the Sanger sequencing technology whose limit of detection are viral populations occurring at $20 \%$ or more and have embraced, the newer, more accurate NGS technology whose limit of detection goes as low as $1 \%$ of the viral populations. With this kind of technology, minority variants are detected much earlier before they evolve to become the dominant variants. At initiation of treatment, if such variants are detected, then the patient is started on the best regimen based on the drug resistance profile. Since HIV drug resistance tests are quite expensive, this precludes baseline drug resistance testing from the standard of care guidelines for management of newly diagnosed HIV patients in the low income countries. Hence, in the developing countries, HIV drug resistance testing is used as a tool for monitoring response to therapy. The advantage of NGS done either at initiation of treatment or during monitoring is that: 1 ). If a patient is found to harbor viral populations that confer drug resistance even at a low level, then they are not initiated on a regimen that they are likely fail on within a short time. 2). If the minority variants are detected during a monitoring visit, their early detection helps in faster decision making so that ultimately these do not evolve to cause cross resistance to other drugs within the same class. Hence multidrug resistance is arrested much earlier therefore some drugs still remain beneficial to the patient especially in the developing countries where there is a limitation on the drug choices to be used.

The purpose of this analysis was twofold: 1 ). To assess the prevalence of drug resistance mutations that are associated with multidrug resistance in patients failing on a RTI regimen. 2). Using NGS, to determine the prevalence of low frequency drug resistance mutations that confer multidrug resistance to patients failing an RTI based regimen. In this analysis, we found that there were definite subtype specific differences with regard to the drug resistance mutations found in patients on ART. Most of these have been reported by others. First, for patients failing on an NNRTI based regimen, K101 which confers high level resistance to Efavirenz (EFV), Nevirapine (NVP) and Etravirine (ETR); and Y181 which confers high level resistance to NVP and ETR although present in the other two subtypes, they occurred at higher proportions in patients harboring subtype $C$ variants. This was also reported by Kiyo et al [27]. This same finding was documented by Neogi et al [71]. Furthermore, the V106 mutation which confers intermediate to high level resistance to EFV and NVP and intermediate level resistance to ETR when present with other mutations in addition to being almost absent in patients harboring subtype $D$ variants, it occurred in such high proportions among patients harboring subtype $C$ HIV variants although Kityo et al [27] did not find a significant difference. The V106 mutation among subtype C HIV variants was first reported by Brenner et al, and Wainberg $[72,73]$. The occurrence of TAMS was significantly less frequent among subtype $C$ than subtype and $D$. The same finding was observed when subtype $C$ was compared to subtype $B$ [74]. On the other hand, the Q151 complex that involves mutations in multiple domains and is notorious for knocking 
off all known NRTIs was significantly more prevalent among subtype $C$ than $A$ and D counterparts. Again, this was reported by Kityo et al [27]. It is important to note that all the mutation domains associated with this complex were significantly much higher in subtype $C$ than the $A$ and $D$ counterparts.

Due to the limit of detection by the Sanger sequencing technology, utilization of NGS with a limit of detection of as low as $1 \%$ of the population enabled us to dissect deeper into these patients to see what is actually happening at levels below the Sanger technology limit of detection. It was very evident that in both patient groups, those who exhibited mutations that confer resistance and those who exhibited a susceptible genotype, HIV drug resistant variants at levels less than $20 \%$ were present. Furthermore, a deep analysis of the TAMs and the Q151 mutation complex revealed that variants that confer multi-drug resistance were present among patients. This finding of the presence of multi-drug resistant variants at low levels within patients either exhibiting MDR or a susceptible genotype has serious clinical implications. Since the HIV genome is constantly evolving under drug pressure, these minority variants will ultimately evolve to become the majority viral populations within the patient thereby influencing response to therapy. However, it is not known how fast these minority variants evolve to become the majority. From these findings it is clear that the presence of a susceptible genotype based on Sanger sequencing does not necessarily preclude presence of drug resistant variants but an indication that more often than not, the virus is evolving but levels below the limit of detection by Sanger. In most of the developing countries a viral load and a drug resistance test are performed once a year as a way of monitoring response to therapy. What this means is that if there were minority variants and a whole year elapses with a patient being kept on a regimen they are silently failing on without close monitoring, full blown multi-drug resistance is inevitable and ultimately this would be evident on Sanger on the next genotype. The danger with this is that patients are unfortunately being kept on regimens they are silently failing on. This may result in transmission of MDR to the new patient and with the test and treat approach that is currently being practiced, a baseline drug resistance test is not performed in resource limited settings hence these patients will be initiated on ART hence the minority drug resistant variants continue to evolve under drug pressure even in the new patients.

It is undeniable that a major hindrance to proper monitoring of HIV patients on ART is the exorbitant costs of HIV drug resistance tests. The emergence of newer technologies have the advantages of not only being able to pick these minority variants at an earlier time point before they cause cross resistance, but also the NGS approach has the advantage of multiplexing such that many more samples can be run thereby cutting the cost per sample. With these new NGS technology therefore, there is hope for reducing the cost per sample while at the same time providing more accurate real time virus evolution.

Therefore, based on the findings of this analysis, we strongly recommend the use of NGS in the resource limited settings as the main platform for HIV drug resistance monitoring since it not only has the ability of reducing the cost per sample but it has the ability to catch mutations as soon as they emerge.

\section{Conclusion}


Although HIV-1 multi drug resistant variants occur in all subtypes, their predominance is subtype specific with TAMs being significantly more predominant among subtype A and D while the Q151M complex significantly more predominant among patients harboring subtype $C$ viruses. Even in patients with a susceptible genotype based on Sanger technology, minority variants are present and their evolution to full blown MDR occurs over time such that by the time they are detectable, cross resistance to other drugs has occurred in some cases.

\section{List Of Abbreviations}

ART Antiretroviral therapy

AZT Azidothymidine

d4T Stavudine

HIV Human immunodeficiency virus

JCRC Joint Clinical Research Center

MDR Multi drug resistance

NGS Next generation sequencing

NNRTI Non-Nucleoside reverse transcriptase inhibitor

NRTI Nucleoside reverse transcriptase inhibitor

PCR Polymerase chain reaction

RT Reverse transcriptase

RTI Reverse transcriptase inhibitor

RT-PCR Reverse transcriptase-Polymerase chain reaction

TAMs Thymidine analog mutations

\section{Declarations}

\section{Ethics approval and consent to participate}

Ethical clearance for this analysis was provided by the institutional review board (protocol, EM-10-07) at JCRC, Kampala Uganda

Consent for publication 
Not applicable

\section{Availability of data and materials}

All data generated or analyzed during this study are included in this published article [and its supplementary information files]

\section{Competing interests}

The authors declare that they have no competing interests

\section{Funding}

Case Western Reserve University Centre for AIDS Research (CWRU/CFAR) through the CFAR Developmental Fund supported this research $v \mathrm{~b}$

\section{Authors' contributions}

This work was carried out in collaboration between all authors. Immaculate Nankya (IN) designed the study, performed data analysis and wrote the manuscript; Eva Nabulime (EN) performed all PCR and Sanger sequencing procedures; Fred Kyeyune performed all the NGS work; Miguel Quinones (MQ) did all the NGS data analysis and Cissy Kityo (CK) made edits and gave comments on the draft. All authors read and approved the final manuscript.

\section{Acknowledgements}

We would like to appreciate the Case Western Reserve University Centre for AIDS Research (CWRU/CFAR) for funding this research through the CFAR developmental fund

\section{References}

1. Wise, J., Global effort to reduce new HIV infections is stalling, UN warns. BMJ, 2019. 366: p. 14744.

2. Duncombe, C., S. Ravishankar, and J.M. Zuniga, Fast-Track Cities: striving to end urban HIV epidemics by 2030. Curr Opin HIV AIDS, 2019. 14(6): p. 503-508.

3. Skeen, S., et al., What will it really take to end the HIV epidemic? AIDS Care, 2018. 30(sup2): p. 1-4.

4. Bain, L.E., C. Nkoke, and J.J.N. Noubiap, UNAIDS 90-90-90 targets to end the AIDS epidemic by 2020 are not realistic: comment on "Can the UNAIDS 90-90-90 target be achieved? A systematic analysis of national HIV treatment cascades". BMJ Glob Health, 2017. 2(2): p. e000227.

5. Nyagah, L.M., et al., HIV-Related Deaths in Nairobi, Kenya: Results From a HIV Mortuary Surveillance Study, 2015. J Acquir Immune Defic Syndr, 2019. 81(1): p. 18-23. 
6. Kandasami, S., et al., Can Changes in Service Delivery Models Improve Program Quality and Efficiency? A Closer Look at HIV Programs in Kenya and Uganda. J Acquir Immune Defic Syndr, 2019. 81(5): p. 533539.

7. Zhang, N., et al., [Impact of achievement of "90-90-90" goals on reduction in new HIV infections in Shandong province based on risk estimation equation]. Zhonghua Liu Xing Bing Xue Za Zhi, 2020. 41(9): p. 1499-1503.

8. Whittaker, R., et al., Monitoring progress towards the first UNAIDS 90-90-90 target in key populations living with HIV in Norway. BMC Infect Dis, 2020. 20(1): p. 451.

9. Chamie, G., et al., Reaching 90-90-90 in rural communities in East Africa: lessons from the Sustainable East Africa Research in Community Health Trial. Curr Opin HIV AIDS, 2019. 14(6): p. 449-454.

10. Porter, K., et al., Substantial Heterogeneity in Progress Toward Reaching the 90-90-90 HIV Target in the WHO European Region. J Acquir Immune Defic Syndr, 2018. 79(1): p. 28-37.

11. Granich, R., et al., Status and methodology of publicly available national HIV care continua and 90-9090 targets: A systematic review. PLoS Med, 2017. 14(4): p. e1002253.

12. Marzel, A., et al., HIV-1 Transmission During Recent Infection and During Treatment Interruptions as Major Drivers of New Infections in the Swiss HIV Cohort Study. Clin Infect Dis, 2016. 62(1): p. 115-122.

13. Soriano, V., The Source of New HIV Infections are People not being Treated or Unaware of their Status. AIDS Rev, 2019. 21(2): p. 108-109.

14. Baggaley, R.F. and T.D. Hollingsworth, How universal does universal test and treat have to be? Lancet HIV, 2020. 7(5): p. e306-e308.

15. Fiorentino, M., et al., Early ART Initiation Improves HIV Status Disclosure and Social Support in People Living with HIV, Linked to Care Within a Universal Test and Treat Program in Rural South Africa (ANRS 12249 TasP Trial). AIDS Behav, 2020.

16. Havlir, D., et al., What do the Universal Test and Treat trials tell us about the path to HIV epidemic control? J Int AIDS Soc, 2020. 23(2): p. e25455.

17. Kiwuwa-Muyingo, S., et al., Lessons for test and treat in an antiretroviral programme after decentralisation in Uganda: a retrospective analysis of outcomes in public healthcare facilities within the Lablite project. Int Health, 2020. 12(5): p. 429-443.

18. Mastro, T.D., M. Bateganya, and H. Mahler, The need to optimize HIV test and treat in Africa. J Infect Dis, 2021. 
19. Mayanja, Y., et al., 'Test and Treat' Among Women at High Risk for HIV-infection in Kampala, Uganda: Antiretroviral Therapy Initiation and Associated Factors. AIDS Behav, 2018. 22(3): p. 1053-1061.

20. Shanaube, K., et al., HIV Care Cascade Among Adolescents in a "Test and Treat" Community-Based Intervention: HPTN 071 (PopART) for Youth Study. J Adolesc Health, 2021. 68(4): p. 719-727.

21. Zhao, Y., J.M. McGoogan, and Z. Wu, The Benefits of Immediate ART. J Int Assoc Provid AIDS Care, 2019. 18: p. 2325958219831714.

22. Ford, N., et al., Benefits and risks of rapid initiation of antiretroviral therapy. AIDS, 2018. 32(1): p. 1723.

23. Kubheka, S.E., M. Archary, and K.K. Naidu, HIV viral load testing coverage and timeliness after implementation of the wellness anniversary in a paediatric and adolescent HIV clinic in KwaZulu-Natal, South Africa. South Afr J HIV Med, 2020. 21(1): p. 1016.

24. Sunpath, H., et al., Targeting the third ' 90 ': introducing the viral load champion. Public Health Action, 2018. 8(4): p. 225-231.

25. AL, D.M. and M.H. Rigatto, Epidemiology of transmitted drug resistance mutations in an HIV-1 subtype C high-prevalence setting and impact on 1-year virological failure. J Glob Antimicrob Resist, 2020. 20: p. 33-35.

26. Omooja, J., et al., Rates of HIV-1 virological suppression and patterns of acquired drug resistance among fisherfolk on first-line antiretroviral therapy in Uganda. J Antimicrob Chemother, 2019. 74(10): p. 3021-3029.

27. Kityo, C., et al., HIV Drug Resistance Mutations in Non-B Subtypes After Prolonged Virological Failure on NNRTI-Based First-Line Regimens in Sub-Saharan Africa. J Acquir Immune Defic Syndr, 2017. 75(2): p. e45-e54.

28. Boender, T.S., et al., Accumulation of HIV-1 drug resistance after continued virological failure on firstline ART in adults and children in sub-Saharan Africa. J Antimicrob Chemother, 2016. 71(10): p. 2918-27.

29. Torres, T.S., et al., Poor quality of life and incomplete self-reported adherence predict second-line ART virological failure in resource-limited settings. AIDS Care, 2021: p. 1-10.

30. Ware, N.C., et al., Influences on Adherence to Antiretroviral Therapy (ART) in Early-Stage HIV Disease: Qualitative Study from Uganda and South Africa. AIDS Behav, 2020. 24(9): p. 2624-2636.

31. Shanmukhappa, S.C., et al., What influences adherence among HIV patients presenting with first-line antiretroviral therapy failure (ART failure)? A retrospective, cross-sectional study from a private clinic in Nagpur, India. J Family Med Prim Care, 2020. 9(12): p. 6217-6223. 
32. Atanga, P.N., et al., Using a composite adherence tool to assess ART response and risk factors of poor adherence in pregnant and breastfeeding HIV-positive Cameroonian women at 6 and 12 months after initiating option B. BMC Pregnancy Childbirth, 2018. 18(1): p. 418.

33. Piana, C., M. Danhof, and O. Della Pasqua, Impact of disease, drug and patient adherence on the effectiveness of antiviral therapy in pediatric HIV. Expert Opin Drug Metab Toxicol, 2017. 13(5): p. 497511.

34. Soboka, M. and G.T. Feyissa, The effectiveness of counseling, material support and/or nutritional supplementation on improving adherence to anti-retroviral therapy and clinical outcomes among HIV patients: a systematic review of quantitative evidence protocol. JBI Database System Rev Implement Rep, 2015. 13(7): p. 142-52.

35. Hardon, A.P., et al., Hunger, waiting time and transport costs: time to confront challenges to ART adherence in Africa. AIDS Care, 2007. 19(5): p. 658-65.

36. Tekin, D., et al., Investigation of drug resistance against protease, reverse transcriptase, and integrase inhibitors by next-generation sequencing in HIV-positive patients. J Med Virol, 2020.

37. Segujja, F., et al., High Levels of Acquired HIV Drug Resistance Following Virological Nonsuppression in HIV-Infected Women from a High-Risk Cohort in Uganda. AIDS Res Hum Retroviruses, 2020. 36(9): p. 782-791.

38. Obasa, A.E., et al., Drug Resistance Mutations Against Protease, Reverse Transcriptase and Integrase Inhibitors in People Living With HIV-1 Receiving Boosted Protease Inhibitors in South Africa. Front Microbiol, 2020. 11: p. 438.

39. Etta, E.M., et al., High level of HIV-1 drug resistance mutations in patients with unsuppressed viral loads in rural northern South Africa. AIDS Res Ther, 2017. 14(1): p. 36.

40. Singh, K., et al., Drug resistance in non-B subtype HIV-1: impact of HIV-1 reverse transcriptase inhibitors. Viruses, 2014. 6(9): p. 3535-62.

41. El-Khatib, Z., et al., Drug resistance patterns and virus re-suppression among HIV-1 subtype $C$ infected patients receiving non-nucleoside reverse transcriptase inhibitors in South Africa. J AIDS Clin Res, 2011. 2(117).

42. Kantor, R. and D. Katzenstein, Drug resistance in non-subtype B HIV-1. J Clin Virol, 2004. 29(3): p. 1529.

43. Gunthard, H.F. and A.U. Scherrer, HIV-1 Subtype C, Tenofovir, and the Relationship With Treatment Failure and Drug Resistance. J Infect Dis, 2016. 214(9): p. 1289-1291. 
44. Kyeyune, F., et al., Treatment failure and drug resistance is more frequent in HIV-1 subtype $D$ versus subtype A-infected Ugandans over a 10-year study period. AIDS, 2013. 27(12): p. 1899-909.

45. Barth, R.E., et al., Accumulation of drug resistance and loss of therapeutic options precede commonly used criteria for treatment failure in HIV-1 subtype-C-infected patients. Antivir Ther, 2012. 17(2): p. 377-86.

46. Soriano, V., Transmission of Multi-Drug Resistant HIV-1 Despite Antiretroviral Prophylaxis. AIDS Rev, 2017. 19(1): p. 54-55.

47. Engchanil, C., et al., Multi-drug resistant HIV-1 reverse transcriptase genotype in children treated with dual nucleoside reverse transcriptase inhibitors (NRTIs). J Med Assoc Thai, 2006. 89(10): p. 1713-20.

48. Takou, D., et al., HIV-1 drug resistance testing is essential for heavily-treated patients switching from first- to second-line regimens in resource-limited settings: evidence from routine clinical practice in Cameroon. BMC Infect Dis, 2019. 19(1): p. 246.

49. Omrani, A.S. and D. Pillay, Multi-drug resistant HIV-1. J Infect, 2000. 41(1): p. 5-11.

50. Miller, M.D., K65R, TAMs and tenofovir. AIDS Rev, 2004. 6(1): p. 22-33.

51. Parikh, U.M., et al., The K65R mutation in human immunodeficiency virus type 1 reverse transcriptase exhibits bidirectional phenotypic antagonism with thymidine analog mutations. J Virol, 2006. 80(10): p. 4971-7.

52. Parikh, U.M., et al., Molecular mechanisms of bidirectional antagonism between K65R and thymidine analog mutations in HIV-1 reverse transcriptase. AIDS, 2007. 21(11): p. 1405-14.

53. Ross, L.L., et al., Differential impact of thymidine analogue mutations on emtricitabine and lamivudine susceptibility. J Acquir Immune Defic Syndr, 2006. 43(5): p. 567-70.

54. Boyer, P.L., et al., Analysis of the Zidovudine Resistance Mutations T215Y, M41L, and L210W in HIV-1 Reverse Transcriptase. Antimicrob Agents Chemother, 2015. 59(12): p. 7184-96.

55. Menendez-Arias, L., Mechanisms of resistance to nucleoside analogue inhibitors of HIV-1 reverse transcriptase. Virus Res, 2008. 134(1-2): p. 124-46.

56. Gibson, R.M., et al., Sensitive detection of HIV-1 resistance to Zidovudine and impact on treatment outcomes in low-to middle-income countries. Infect Dis Poverty, 2017. 6(1): p. 163.

57. De Luca, A., et al., Accumulation of HIV-1 drug resistance in patients on a standard thymidine analogue-based first line antiretroviral therapy after virological failure: implications for the activity of next-line regimens from a longitudinal study in Mozambique. BMC Infect Dis, 2017. 17(1): p. 605.

58. Andreoletti, L., et al., Multidrug-resistant HIV-1 RNA and proviral DNA variants harboring new dipeptide insertions in the reverse transcriptase pol gene. J Acquir Immune Defic Syndr, 2002. 29(1): p. 102-4. 
59. Bulgheroni, E., et al., Unusual codon 69 insertions: influence on human immunodeficiency virus type 1 reverse transcriptase drug susceptibility. J Clin Virol, 2004. 29(1): p. 27-32.

60. Kaliki, V., et al., Emergence of HIV-1 variants containing codon insertions and deletions in the beta3beta4 hairpin loop domain of reverse transcriptase. Immunol Lett, 2000. 74(2): p. 173-5.

61. Menendez-Arias, L., T. Matamoros, and C.E. Cases-Gonzalez, Insertions and deletions in HIV-1 reverse transcriptase: consequences for drug resistance and viral fitness. Curr Pharm Des, 2006. 12(15): p. 181125.

62. Quinones-Mateu, M.E., et al., Insertions in the reverse transcriptase increase both drug resistance and viral fitness in a human immunodeficiency virus type 1 isolate harboring the multi-nucleoside reverse transcriptase inhibitor resistance 69 insertion complex mutation. J Virol, 2002. 76(20): p. 10546-52.

63. Tamalet, C., et al., Multidrug resistance genotypes (insertions in the beta3-beta4 finger subdomain and MDR mutations) of HIV-1 reverse transcriptase from extensively treated patients: incidence and association with other resistance mutations. Virology, 2000. 270(2): p. 310-6.

64. Marcelin, A.G., et al., Thymidine analogue reverse transcriptase inhibitors resistance mutations profiles and association to other nucleoside reverse transcriptase inhibitors resistance mutations observed in the context of virological failure. J Med Virol, 2004. 72(1): p. 162-5.

65. Mbisa, J.L., et al., The evolution of HIV-1 reverse transcriptase in route to acquisition of Q151M multidrug resistance is complex and involves mutations in multiple domains. Retrovirology, 2011. 8: p. 31.

66. Emergence of M184V mutation. AIDS Patient Care STDS, 2004. 18(8): p. 488.

67. Gallant, J.E., The M184V mutation: what it does, how to prevent it, and what to do with it when it's there. AIDS Read, 2006. 16(10): p. 556-9.

68. Olearo, F., et al., Impact of the M184V/I Mutation on the Efficacy of Abacavir/Lamivudine/Dolutegravir Therapy in HIV Treatment-Experienced Patients. Open Forum Infect Dis, 2019. 6(10): p. ofz330.

69. Turner, D., B. Brenner, and M.A. Wainberg, Multiple effects of the $M 184 \mathrm{~V}$ resistance mutation in the reverse transcriptase of human immunodeficiency virus type 1. Clin Diagn Lab Immunol, 2003. 10(6): p. 979-81.

70. Gibson, R.M., C.L. Schmotzer, and M.E. Quinones-Mateu, Next-Generation Sequencing to Help Monitor Patients Infected with HIV: Ready for Clinical Use? Curr Infect Dis Rep, 2014. 16(4): p. 401.

71. Neogi, U., et al., Selection of nonnucleoside reverse transcriptase inhibitor-associated mutations in HIV-1 subtype C: evidence of etravirine cross-resistance. AIDS, 2011. 25(8): p. 1123-6. 
72. Brenner, B., et al., A V106M mutation in HIV-1 clade $C$ viruses exposed to efavirenz confers crossresistance to non-nucleoside reverse transcriptase inhibitors. AIDS, 2003. 17(1): p. F1-5.

73. Wainberg, M.A., HIV-1 subtype distribution and the problem of drug resistance. AIDS, 2004. 18 Suppl 3: p. S63-8.

74. Koning, F.A., et al., Subtype-specific differences in the development of accessory mutations associated with high-level resistance to HIV-1 nucleoside reverse transcriptase inhibitors. J Antimicrob Chemother, 2013. 68(6): p. 1220-36.

\section{Figures}




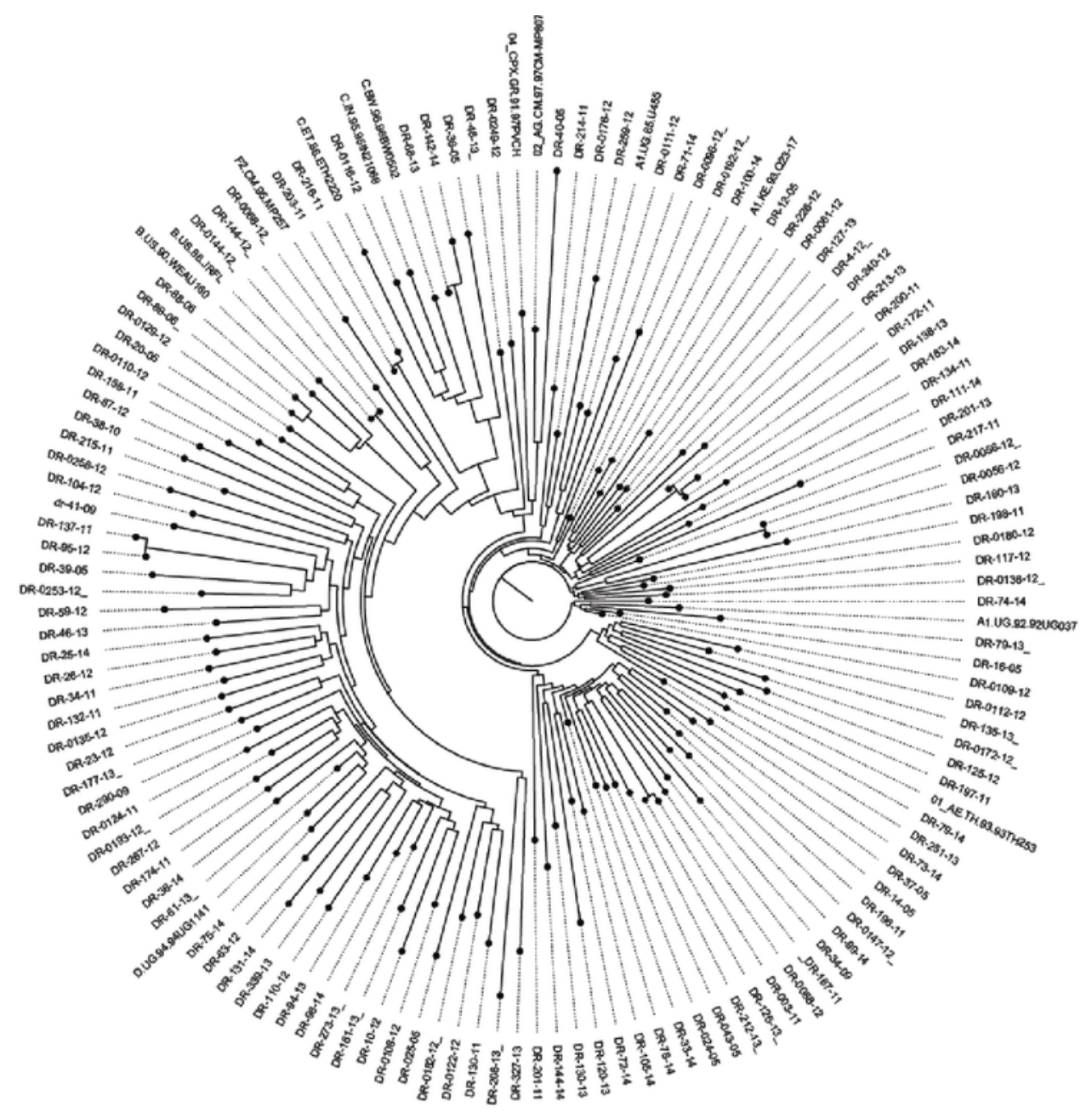

\section{Figure 1}

Phylogenetic tree showing subtype distribution among patients failing an NRTI based regimen. Phylogenetic tree was constructed for all patients failing on an NRTI based regimen by compiling all the RT sequences obtained by Sanger sequencing. Sequences were aligned against known subtype references. 

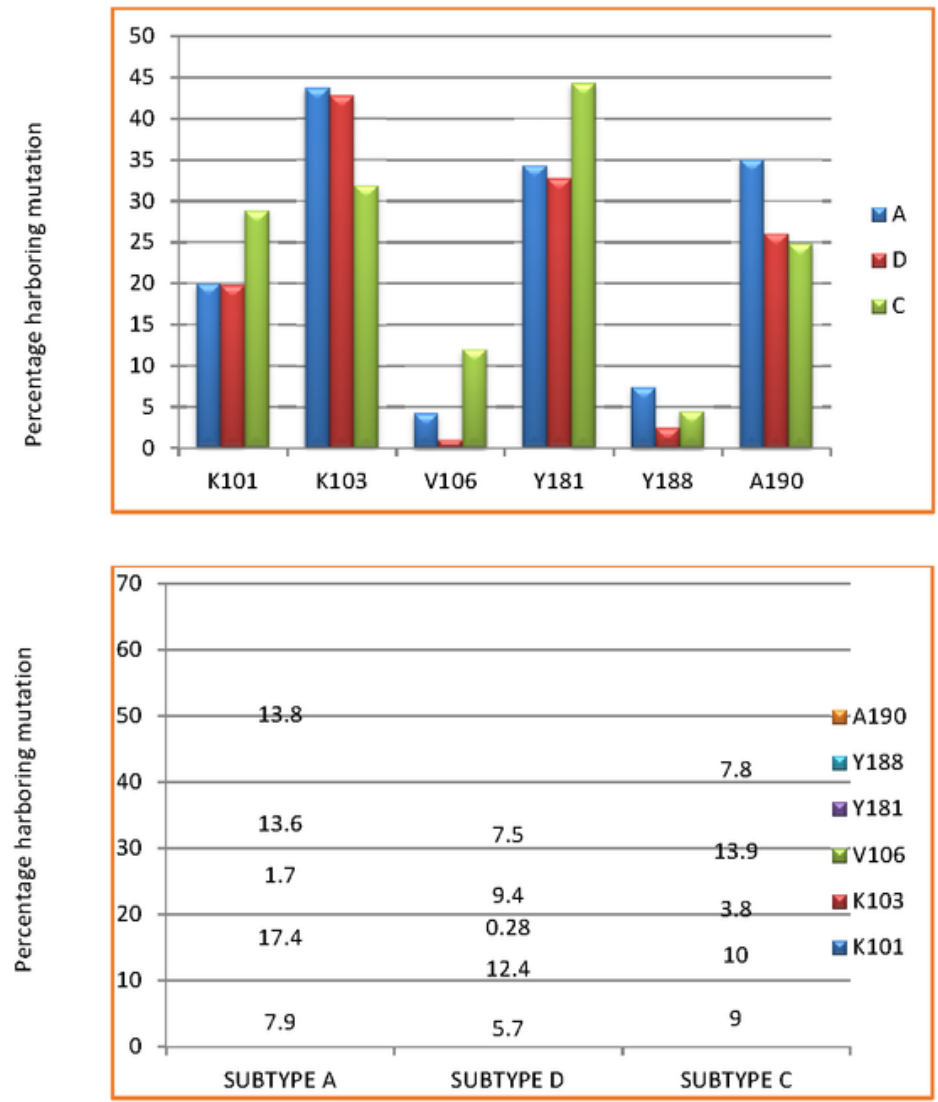

\section{Figure 2}

Mutations that confer resistance to NNRTIs by subtype. For all the patients on an NNRTI based regimen, known mutations that confer resistance to NNRTIs were analyzed by subtype to see if there were any subtype differences. Percentage at individual codons by subtype (Fig 2A) and overall percentage by subtype was analyzed using Sanger sequencing technology (figure 2B) 


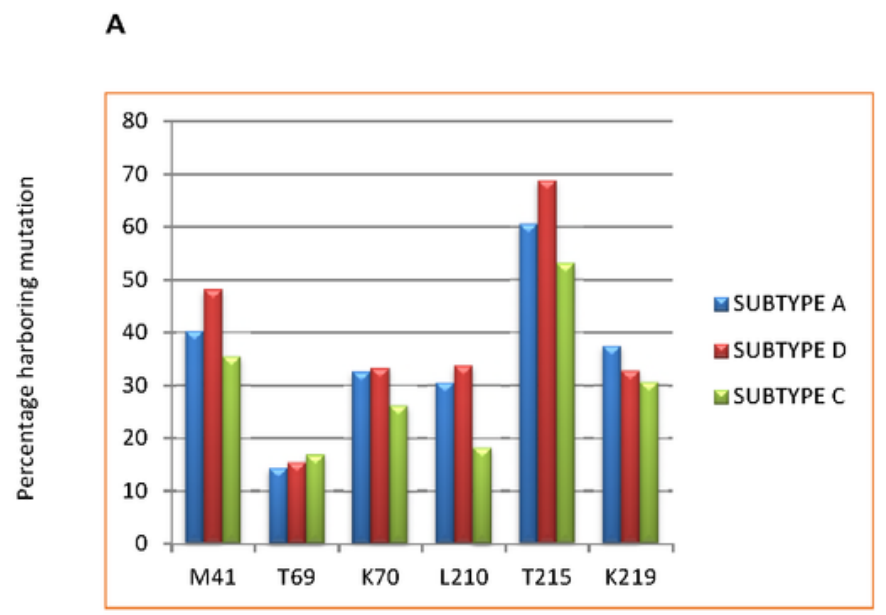

B

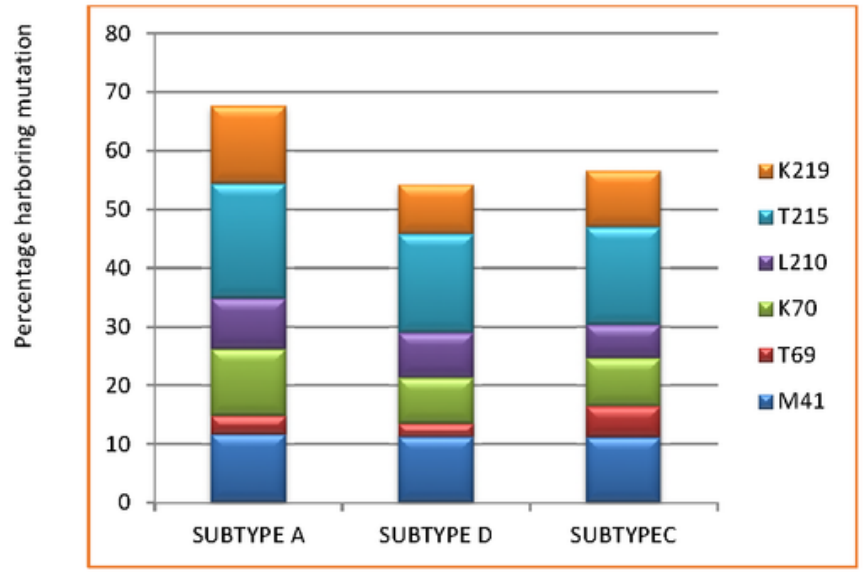

Figure 3

TAMS Multidrug resistance by subtype. For all patients on an NRTI based regimen, thymidine analog mutations (TAMS) were analyzed for subtype A, C and D. Level of resistance at individual codons (Fig 3A) and overall resistance (fig $3 \mathrm{~B}$ ) performed by subtype using Sanger sequencing technology is shown. 


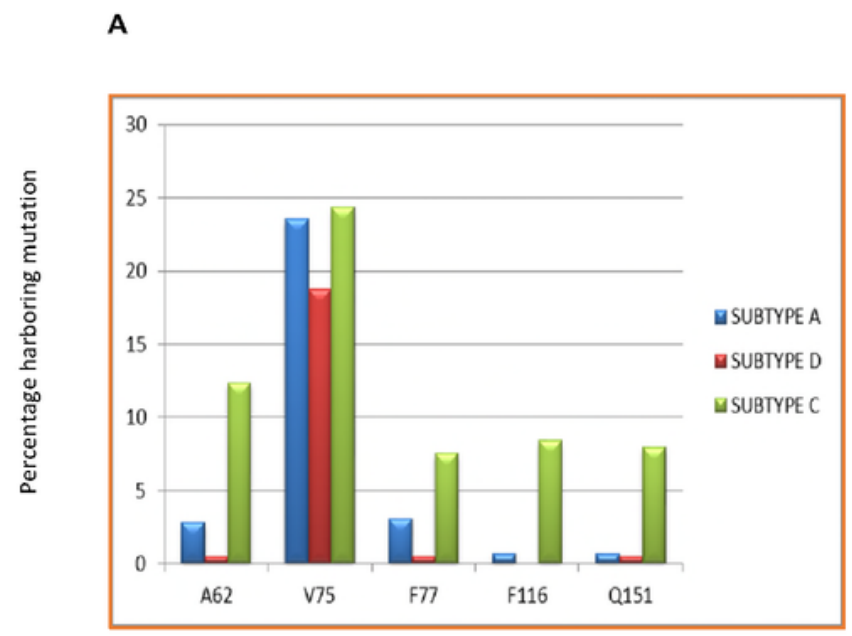

B

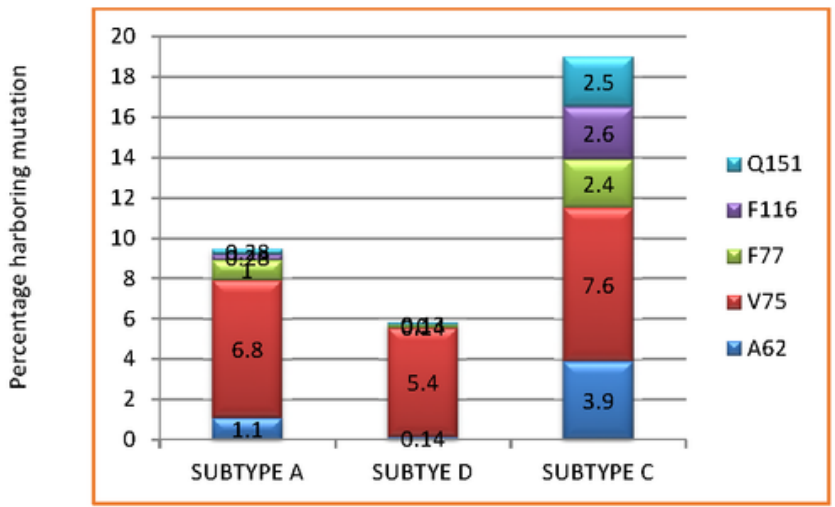

Figure 4

Q151 complex multidrug resistance by subtype. For patients failing on an NRTI based regimen, Q151 associated mutations were analyzed for subtype $A C$ and D. Level of resistance at individual codons (Fig 4A) and overall resistance (Fig 4B) by subtype performed using Sanger sequencing technology is shown. 

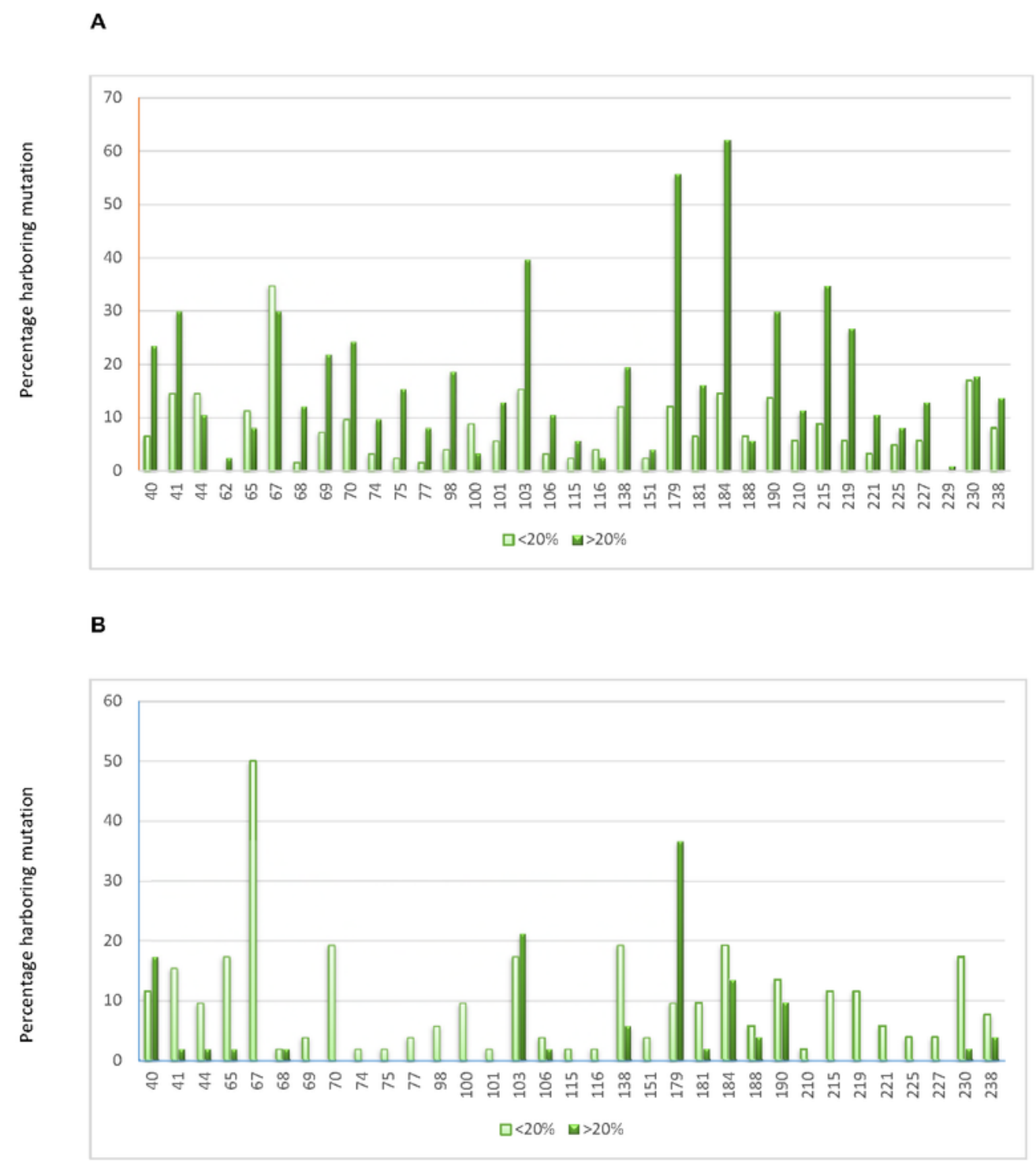

\section{Figure 5}

Drug resistance mutations in the RT coding region. Drug resistance mutations were assayed for both NRTIs and NNRTIs by codon using Next Generation sequencing. Mutation profiles were analyzed at a $20 \%$ cutoff and at $<20 \%$ in order to assess the prevalence of low level mutation profiles that are present below the $20 \%$ level. Low level mutation profiles were analyzed for patients exhibiting drug resistance mutations 
by Sanger (Fig 5A); low level mutation profiles were analyzed for patients susceptible profile based on Sanger (Fig 5B).
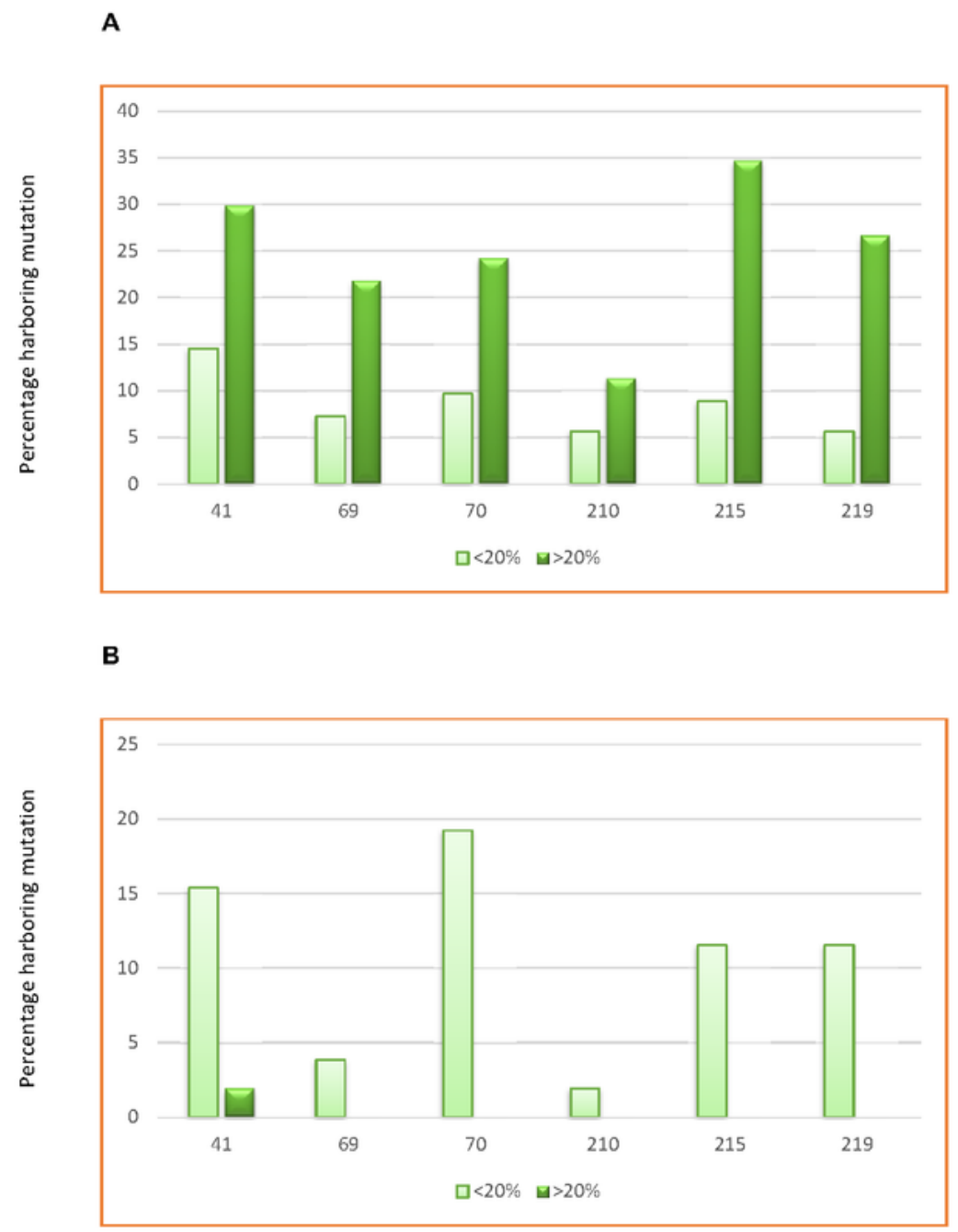

Figure 6

TAMs in patients failing an NRTI based regimen. Using Next Generation Sequencing, presence of TAMs was analyzed by codon for patients failing an NRTI based regimen. Presence of TAMs was assessed at 
$>20 \%$ and $<20 \%$ levels in patients who had drug resistance mutations (Fig $6 \mathrm{~A}$ ) and in patients who failed but with a susceptible genotype (Fig 6B).
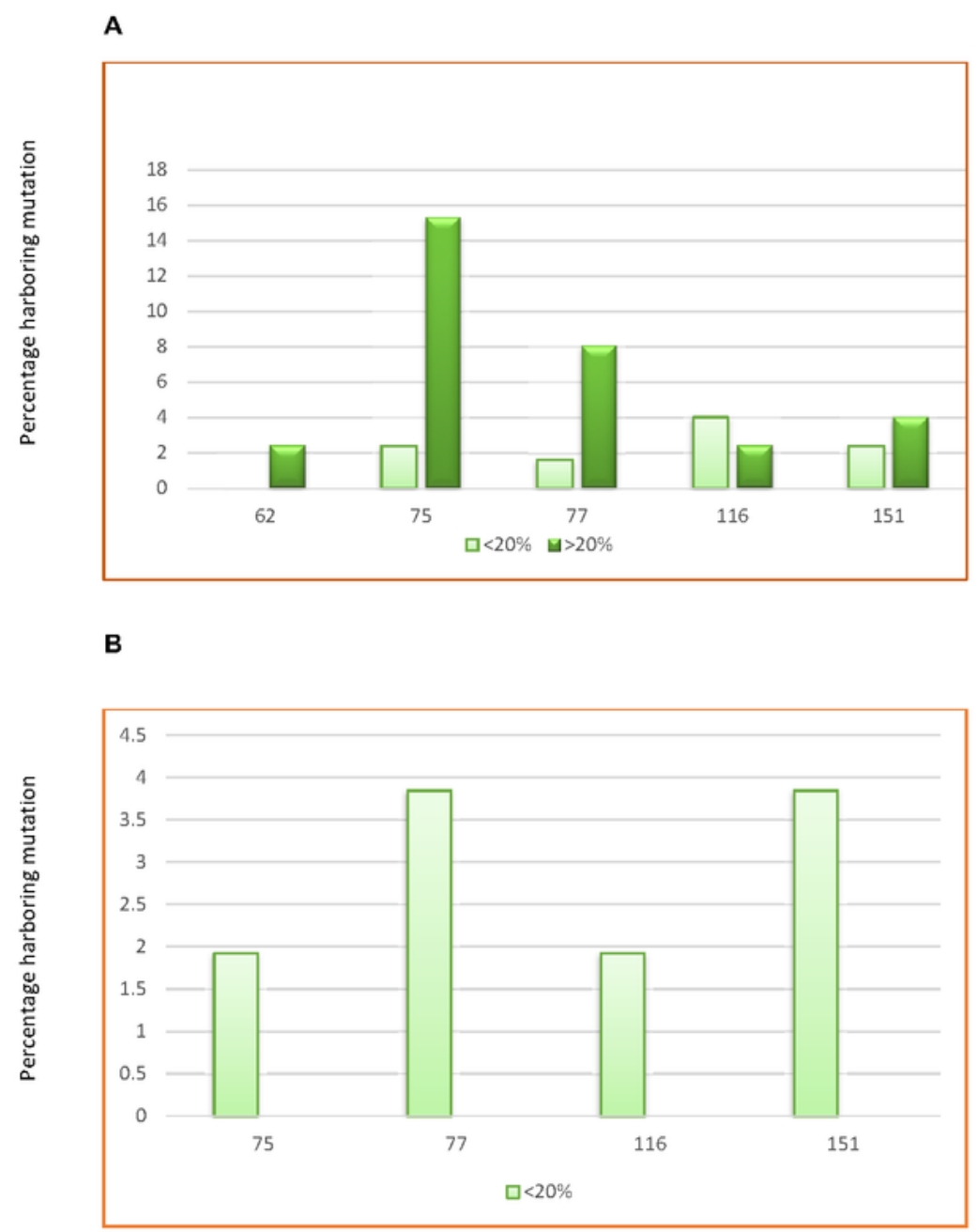

Figure 7

Q151 complex in patients failing an NRTI based regimen. Using Next Generation Sequencing, presence of the Q151 mutation complex that confer drug resistance to all known NRTIs was analyzed by codon for patients failing an NRTI based regimen. Presence of the Q151 complex was assessed at $>20 \%$ and $<20 \%$ 
levels in patients who had drug resistance mutations (Fig 7A) and in patients who failed but with a susceptible genotype (Fig 7B). 